\title{
OPTICAL FIBRE SENSORS AND METHODS
}

\author{
Manish Munjal ${ }^{1 *}$, Amit Grewal ${ }^{2}$, Harsh Yadav ${ }^{3}$ \\ ${ }^{* 1}$ Btech (Student), ${ }^{2}$ Btech (Student), ${ }^{3}$ Btech (Student) \\ *1,2,3 Department Of Electronics and Communication Engineering, Dronacharya College Of Engineering, Gurgaon \\ ${ }^{* 1}$ Email ID: manishmunjal94@gmail.com
}

*Corresponding Author: -

Email: manishmunjal94@gmail.com

\begin{abstract}
:
This research paper is about a multi-mode fibre optic sensor for optically sensing a physical perturbation including a multi- mode optical fibre segment which accepts coherent monochromatic radiation from a suitable source. As the radiation is propagated in the fibre, the various modes form a complex interference pattern which changes in response to a physical perturbation of the fibre. A detector provides an output signal to a signal processor which analyzes the signal as a function of the change in intensity to provide an information signal functionally related to the perturbation.
\end{abstract}

Keywords: $C C D$

\section{(c) \$ (\$)}




\section{INTRODUCTION}

Optical energy transmitted through the core of an optical fibre, either a single or multi-mode core, is affected by physical perturbations of the fibre. Typically, the physical perturbation will alter the index of refraction of the core material as well as the differential indices between the cladding and the core in such a way that the optical energy transmitted through the core is changed. The physical perturbation can be caused, for example, by strain induced by tension or compression as well as strain induced by bending the fibre about a small radius (i.e., micro-bending) or large radius bending (i.e., macro-bending). Accordingly, an optical fibre can be used as a sensor to measure a physical parameter by correlating changes in the output energy with the environmental perturbations. The energy output from the sensing fibre can be analyzed, for example, in terms of quantitative changes in intensity, wavelength, or polarization states. In a more sophisticated context, the output light can be interierometrically compared against a reference source to provide an interferometer pattern which can be empirically correlated with the fibre-perturbing parameter. In the interferometric context, e.g., a Mach-Zender interferometer, coherent source light is passed through reference and sensing fibres with the light from the two paths combined to form an interferometric pattern which is analyzed to provide information functionally related to an external perturbation affecting the sensing fibre path. Optical fibre sensing systems have a number of applications, especially in the aerospace field. For example, the wing or tail section of an aircraft can be 'fibred' and the output light analyzed to determine stress levels in the structure as well as the presence of pre-fracture conditions or the presence of fractures caused by fatigue or ballistic impact. Optical fibre sensing systems have the advantage of an exceptionally wide bandwidth so that the fibre can serve both a sensing function and a data transmission function.

\section{Diagram}

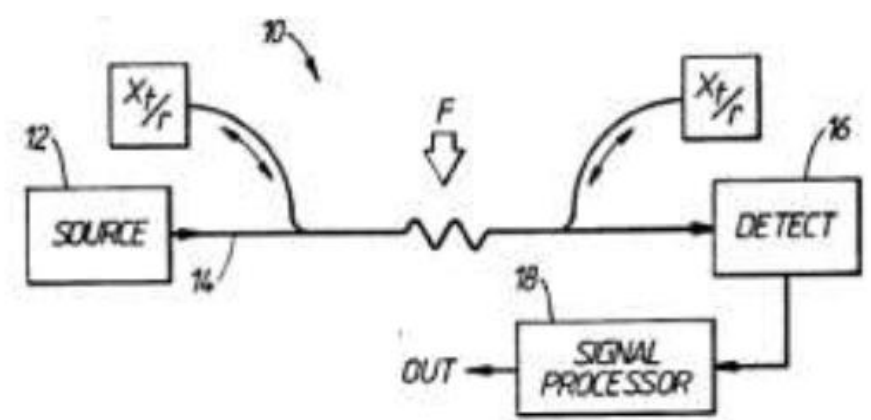

Fig: Optical Fibre Sensor

\section{Working}

There is provided a multi-mode fibre optic sensor responsive to a physical perturbation, characterised by a multi-mode optical fibre for transmitting optical energy between an input and an output in at least two modes to form an interference pattern between the modes, means for providing optical energy to the input of said optical fibre sufficient to propagate at least the said two modes along the fibre such that the interference pattern changes in response to a physical perturbation of the fibre, and means for detecting an interference pattern from the output of said optical fibre and generating a signal functionally related to the perturbation and in response to an intensity variation of at least a part of the interference pattern. More specifically there will be described a multi- mode optical fibre sensor and method in which coherent monochromatic radiation from an optical energy source passes through a multi-mode optical fibre which is subjected to an external per-turbation.

As the light is conducted through the core, the various modes constructively and de- structively interfere with one another with the pro- jected output having a characteristic inter-modal "speckle" pattern. A detector, such as a two dimensional staring array, outputs an electrical array signal in response to the intensity distribution of the speckle pattern. As the fibre or a segment thereof is perturbed, the inter-modal interference pattern and the intensity distribution changes in a manner functionally related to the perturbation. The corresponding output of the detector is analyzed by a signal processor to provide a signal output representative of the perturbation.

In a first embodiment, a monochromatic light source, such as a laser diode, inputs coherent light into a multi-mode optical fibre segment which is subject to environmental perturbations. The coher- ent light, as it travels through the core, assumes different modes, including a lowest order and at least one higher order mode. The various modes constructively and destructively interfere to pro- duce a characteristic 'speckle' pattern which is projected through a spatial filter onto a photodetec- tor. In its simplest form, the spatial filter is defined by a light-blocking sheet having one or more ap- ertures that pass a subset of the speckle pattern to the photodetector. The signal output of the photodetector varies in response to the variation in the intensity distribution of that portion of the speckle pattern passed to the photodetector by the spatial filter.

The output of the photodetector is fed to a signal processor with the change in the intensity distribution functionally related to the sensed per- turbations. In another embodiment the output light from the multi- mode optical fibre is projected onto a multi-pixel CCD array. As the CCD array is scanned, its outputs are sent to a signal processor which converts the individual pixel output into a corresponding digital value and stores the digitized array output as two 
successive data frames. The absolute value of the change between correspond- ing pixel data points in the two data frames is summed to provide a signal output functionally related to the sensed perturbations.

\section{Utility}

The multi-mode optical fibre sensors described use the constructive and destructive interference of coherent light in a multi-mode fibre to provide optical information useful in providing a signal which functionally related to the sensed perturba- tion. The sensors are particularly useful in sensing information relating to stressed structures, such as those in aircraft and other vehicles and well as use as intrusion detection, for example. As will be apparent to those skilled in the art, various changes and modifications may be made to the illustrated multi-mode optical fibre sensors and methods of the present invention without departing from the spirit and scope of the invention as deter- mined in the appended claims and their legal equivalent.

An optical source launches coherent radiation into a single mode optical fibre segment which is coupled at to a multimode optical fibre subjected to the per- turbations to be sensed, thereby reducing error from undesired vibrations. After the desired per- turbation has been sensed by the multi-mode optical fibre segment, the complex interference pattern is output from the multi-mode optical fibre segment through a spatial filter. A subset of the complex interference pattern passes through the spatial filter and is focused through a lens into a multi-mode optical fibre segment. The complex interference pattern is transmitted along the multi-mode optical fibre segment to a photo detector, which outputs a signal in response to the intensity of the subset of the com- plex interference pattern passed by the spatial filter.

\section{Conclusion}

A multi-mode fibre optic sensor responsive to a physical perturbation, characterised by a multi- mode optical fibre for transmitting optical energy between an input and an output in at least two modes to form an interference pattern between the modes, means for pro- viding optical energy to the input of said optical fibre sufficient to propagate at least the said two modes along the fibre such that the interference pattern changes in response to a physical perturbation of the fibre, and means for detecting an interference pattern from the output of said optical fibre and generating a signal functionally related to the perturbation and in response to an intensity variation of at least a part of the interference pattern. The detecting means comprises means for photodetecting a subset of the com plex interference pattern from the output of said optical fibre segment, the photodetecting means generating said signal in response to a variation in the intensity of the subset of the complex interference pattern, and means for filtering disposed between the output and said photodetector means for feeding the sub- set of the complex interference pattern to said photo detector means.

\section{Reference}

[1].Fibre Optics, John M. Senior

[2].Fibre Optics Sensors, Wikipedia 